\title{
ERRATUM: ASYMMETRIC SHAPES OF RADIO RECOMBINATION LINES FROM IONIZED STELLAR WINDS. (RMxAA, 2019, 55, 31)
}

\author{
R. Ignace \\ Department of Physics \& Astronomy, East Tennessee State University.
}

Received January 14 2020; accepted January 172020

A mistake was discovered in part of the derivation for analytic line shapes of radio recombination lines. The mistake does not change the functional form of the solution, but does alter an amplitude factor.

Ignace (2019) considered the analytic derivation for radio recombination lines (RRLs) under simplifying conditions of spherically symmetric, isothermal, and terminal speed flow in which both continuum (free-free) and line (recombination) opacities scale as the square of density. A mistake has been identified in equation (13). A second "formal" error was subsequently identified, but turns out to have no bearing on the derivation, as will be explained.

For context, the solution allowed for a power-law distribution in the clumping parameter:

$$
D_{\mathrm{cl}} \propto r^{-m}
$$

with $m$ being the power-law exponent. In Ignace (2019), the solution for the emergent flux from the wind comes from an analytic integral. As published, equation (13) is

$$
\int_{0}^{\infty}\left(1-e^{-a x^{\beta}}\right) x d x=\frac{1}{\beta} \Gamma\left(\frac{2}{\beta}\right) a^{2 / \beta}
$$

where $\Gamma$ is the Gamma-function. However, the correct expression should be

$$
\int_{0}^{\infty}\left(1-e^{-a x^{-\beta}}\right) x d x=\frac{-1}{\beta} \Gamma\left(\frac{-2}{\beta}\right) a^{2 / \beta},
$$

where minus signs had been dropped by mistake.

While correcting the above mistake, another error was identified. From Ignace (2019), equation (23) correctly provides the following analytic integral:

$$
\int_{0}^{\infty} x^{-\beta} e^{-a x} d x=\Gamma(1-\beta) a^{\beta-1} .
$$

However, the domain of application requires that $\beta>-1$, but such is not the case, with $\beta=-(5+m) /(3+m)$. Application of this lookup integral was intended for the second and third terms of equation (22), for which the combined integral would be of the form

$$
\int_{0}^{\infty}\left(1-e^{-\tau_{L}}\right) e^{-\tau_{W}} p d p
$$

This integral does converge. What the paper does is to split up the integrand terms and apply equation (23) to each. Separately, these integrals formally diverge. However, it has been verified numerically for the examples of $m=0$ and $m=1$ that the correct answer still results. The solution for the flux needs corrected only for the mistake in equation (13).

Ultimately, that mistake propagates into equations (18), (20), (24), and implicitly (27), only in multiplicative coefficients. Equation (27) is corrected by setting $\gamma_{\mathrm{m}}=1$, to give 


$$
\begin{aligned}
\frac{f\left(w_{\mathrm{z}}\right)}{f_{C}}= & {\left[1+\frac{t_{L C}(\lambda)}{G_{\mathrm{m}}(\pi)}(\sin \theta)^{1+m}\right]^{2 /(3+m)} } \\
& +\delta_{L C}\left\{\left[\frac{G_{\mathrm{m}}(\theta)}{G_{\mathrm{m}}(\pi)}+\frac{t_{L C}(\lambda)}{G_{\mathrm{m}}(\pi)}(\sin \theta)^{1+m}\right]^{2 /(3+m)}-\left[\frac{G_{\mathrm{m}}(\theta)}{G_{\mathrm{m}}(\pi)}\right]^{2 /(3+m)}\right\}
\end{aligned}
$$

now with

$$
f_{C}=\pi S_{C}\left(\frac{R_{*}^{2}}{d^{2}}\right) \Gamma\left(\frac{1+m}{3+m}\right)\left[G_{\mathrm{m}}(\pi) \mathcal{T}_{C}(\lambda)\right]^{2 /(3+m)} .
$$

For Figures (2)-(5), the profiles are all correct, but not for the parameters as labeled. For a given profile in Ignace (2019), the mapping from the incorrect parameters (original, or "orig") to the correct parameters ("new") is:

$$
\delta_{L C}^{\text {new }}=\delta_{L C}^{\text {orig }} \gamma_{\mathrm{m}}^{\text {orig }} .
$$

For $m=-0.5,0$, and $1, \gamma_{\mathrm{m}}^{\text {orig }}=12.3,8.9$, and 8.0.

Appreciation is expressed to Ian Stevens for alerting me to the error in the published manuscript. 